\title{
Cryptococcal Meningitis: A Retrospective Cohort of a Brazilian Reference Hospital in the Post-HAART Era of Universal Access
}

\author{
Aline Z. de Azambuja, Gustavo Wissmann Neto, Guilherme Watte, Luciana Antoniolli, \\ and Luciano Z. Goldani iD
}

Section of Infectious Diseases, Hospital de Clínicas de Porto Alegre, Universidade Federal do Rio Grande do Sul, Porto Alegre, RS, Brazil

Correspondence should be addressed to Luciano Z. Goldani; lgoldani@ufrgs.br

Received 9 May 2018; Revised 1 July 2018; Accepted 12 July 2018; Published 1 August 2018

Academic Editor: Aim Hoepelman

Copyright (C) 2018 Aline Z. de Azambuja et al. This is an open access article distributed under the Creative Commons Attribution License, which permits unrestricted use, distribution, and reproduction in any medium, provided the original work is properly cited.

Objective. Cryptococcus neoformans is a common opportunistic infection in adults with acquired immunodeficiency syndrome worldwide. However, limited data exist for HIV-infected patients in the post-HAART (highly active antiretroviral therapy) era in Brazil. The aim of this study was to describe the clinical characteristics and outcomes of cryptococcosis in a cohort of patients attending a teaching tertiary care hospital in southern Brazil after the introduction of HAART in Brazil. Patients and Methods. A retrospective study was conducted in tertiary care hospital in southern Brazil. Detailed data on risk factors, clinical manifestations, diagnosis methods, treatment, and prognosis of patients with meningeal cryptococcosis were evaluated from January 2009 to December 2016. Results. Seventy-nine cases of cryptococcal meningitis were identified. Most of the patients presented positive CSF (cerebrospinal fluid) cultures for Cryptococcus neoformans (96\%). The prevalence of males and females with meningeal cryptococcosis was similar. The age of the patients ranged from 5 to 67 years. The median time of hospitalization was 28 days. The most common underlying disease was HIV (82\%), followed by solid transplant (10\%). Fever, nausea, vomiting, headache, and altered mental status were the most common clinical manifestations. Initial opening intracranial pressures varied from 30 to $130 \mathrm{~cm} \mathrm{H} \mathrm{H}_{2} \mathrm{O}$. CNS imaging abnormalities include hydrocephalus and hypodensities. Widened Virchow-Robin spaces were described in only 2 patients (2.5\%). Induction treatment of the majority of the patients consisted of amphotericin B and flucytosine (67\%) followed by amphotericin B and fluconazole (19\%). Multivariate analysis of Cox regression identified headache at presentation, mechanical ventilation, CSF glucose $<20 \mathrm{mg} / \mathrm{dL}$, and CSF cryptococcal antigen $\geq 1: 1000$ for independent risk factors for death. All-cause 30-day and 60-day mortalities were $19 \%$ and $24 \%$, respectively. Conclusions. Meningeal cryptococcosis mostly caused by C. neoformans continues to occur predominantly in HIV-infected adults despite HAART being widely distributed in Brazil. Cryptococcosis remains a significant opportunistic infection in solid organ transplant recipients. Despite adequate antifungal treatment and management of intracranial hypertension in a reference tertiary care hospital, mortality was high. Identification of risk factors and additional treatment modalities, especially for intracranial hypertension, are necessary to improve care for patients with cryptococcal meningitis.

\section{Introduction}

Cryptococcal meningitis may be the presenting manifestation of AIDS. The most common sites of occurrence of this infection are the central nervous system and the lungs [1-3]. Inhalation of cryptococcal particles from contaminated soil into the lung is considered the usual route of human infection. This organism disseminates hematogenously, and it has a propensity to localize in the central nervous system, causing meningoencephalitis [2]. Although the incidence of cryptococcal meningitis has declined in the HIV patients who undergo antiretroviral therapy, cryptococcal disease remains a leading cause of mortality in the developing world $[4,5]$. The clinical signs and symptoms of C. neoformans meningitis are indistinguishable from those of many other causes of meningitis, especially tubercular meningitis. An 
early diagnosis of the cryptococcal infection is therefore necessary for its appropriate management.

A reduction in the rate of opportunistic infections and hospitalizations in adults infected with AIDS after HAART intervention is well documented in countries with universal access to antiretrovirals [6]. The present work is a retrospective study of patients with cryptococcal meningitis admitted to a Brazilian tertiary care hospital 23 years after the introduction of HAART in Brazil.

\section{Materials and Methods}

This retrospective analysis of epidemiological and clinical data from patients with meningeal cryptococcosis was performed during the period of January 2009 to December 2016 in a tertiary care hospital in southern Brazil. Cryptococcal meningitis was confirmed by a positive CSF cryptococcal culture and/or cryptococcal antigen test. The medical records of all eligible patients were reviewed, and data regarding demographics, medical history, clinical and laboratory characteristics, treatment regimens, and clinical outcomes were collected. Patients were evaluated for risk factors and mortality.

2.1. Statistical Analysis. Data were analyzed using SPSS software, version 18 or superior. Results were expressed in mean and standard deviation or median and interquartile range for variables in numeric scale and number and percentage for variables in nominal scale. Qualitative variables were compared by the chi-square test, Fisher's exact test, or McNemar test, when indicated. For determination of the factors associated with overall, 30- and 60-day mortalities, multivariate logistic regression models were constructed using a stepwise regression. A predictive modeling strategy was used in which variables were selected based on the association with mortality in univariate analysis (using a $p$ value $\leq 0.20$ ) and retained if they significantly improved model fit. The Cox regression model was used for determination of risk factors associated with overall mortality. A Poisson regression model with robust variance was employed for determination of risk factors associated with mortality at 30 and 60 days. The Kaplan-Meier survival method was adopted for survival analysis of cohort. The survival between groups (HIV and non-HIV) was compared by log-rank test. For statistical significance, a $p$ value $\leq 0.05$ was considered.

\section{Results}

As shown in Table 1, seventy-nine cases of cryptococcal meningitis were identified. Most of the patients presented positive CSF cultures for Cryptococcus neoformans (96\%). The prevalence of males and females with meningeal cryptococcosis was similar. The age of the patients ranged from 5 to 67 years. As expected, the mean time of hospitalization was long (28 days). The most common underlying disease was HIV (82\%), followed by solid transplant (10\%). Only $34(43 \%)$ patients were on highly active antiretroviral therapy (HAART). Medication adherence was not measured
TABLE 1: Baseline characteristics of 79 patients with cryptococcal meningitis.

\begin{tabular}{lc}
\hline Variable & \\
\hline Age, years; mean (range) & $37(5-67)$ \\
Age $\geq 50$ years; $N$ (\%) & $17(21.5)$ \\
Sex, male $N$ (\%) & $45(56.9)$ \\
Time of hospitalization,days; mean (range) & $28(1-149)$ \\
Type of immunosuppression & $N(\%)$ \\
HIV & $65(82.3)$ \\
SOTR & $8(10.1)$ \\
NHNT & $6(7.6)$ \\
HIV patients & \\
Dx during hospitalization & \\
Vertical transmission of HIV & $14(17.7)$ \\
Receiving HAART at Dx & $3(3.7)$ \\
CD4, cells/mm ${ }^{3}$ mean (range) & $34(43.0)$ \\
HIV viral load, log; mean (range) & $34(3-428)$ \\
SOTR patients & $5.01(2.18-6.16)$ \\
Kidney & $N(\%)$ \\
Liver & $5(10.1)$ \\
Duration of transplant, years & $3(10.1)$ \\
General; mean (range) & \\
Kidney; mean (range) & $2.12(0-7.35)$ \\
Liver; mean (range) & $3.58(0.25-7.36)$ \\
Other concomitante conditions & $2.12(0-7.35)$ \\
Use of corticosteroids & $N(\%)$ \\
Chronic kidney disease & $8(10.1)$ \\
Cancer & $2(2.5)$ \\
Rheumatic disease & $4(5.1)$ \\
Diabetes mellitus & $2(2.5)$ \\
Cirrhosis & $3(3.8)$ \\
Cryptococcus species complex & $2(2.5)$ \\
C. neoformans & \\
C. gattii & $76(96.2)$ \\
Duration of symptoms, days; mean (range) & $3(3.8)$ \\
Rehospitalization & $10(1-120)$ \\
30-day mortality & $6(7.5)$ \\
60-day mortality & $15(19.1)$ \\
\hline SOTR sold orgat & $19(24.4)$ \\
\hline
\end{tabular}

SOTR, solid organ transplant recipients; NHNT, non-HIV nontransplant; ESRD, end-stage renal disease. ${ }^{a}$ Diagnosis of HIV concomitant with neurocryptococcosis.

in these patients. The vast majority of the patients with meningeal cryptococcosis were infected by Cryptococcus neoformans (96\%). Fever, nausea, vomiting, headache, and altered mental status were the most common manifestations (Table 2). Laboratory findings are described in Table 3. High CSF cryptococcal antigen was present in $60 \%$ of the patients. The majority of the patients developed intracranial hypertension. Initial opening Intracranial pressures varied from 30 to $130 \mathrm{~cm} \mathrm{H}_{2} \mathrm{O}$. Despite appropriate antifungal treatment, patients persisted with intracranial hypertension. The CNS imaging abnormalities include hydrocephalus and hypodensities. The classical finding of widened Virchow-Robin spaces was described in only 2 patients $(2.5 \%)$. Induction treatment of the majority of the patients consisted of amphotericin B and flucytosine (67\%) followed by amphotericin B and fluconazole (19\%) (Table 4). Fluconazole was combined with amphotericin B for some patients because flucytosine was not commercially available in Brazil for a certain period of time. All-cause overall 30-day and 
TABLE 2: Clinical manifestations of 79 patients with cryptococcal meningitis.

\begin{tabular}{lc}
\hline Variable & $N(\%)$ \\
\hline Fever & $43(54.4)$ \\
Malaise & $22(27.8)$ \\
Headache & $58(73.4)$ \\
Nausea and vomiting & $33(41.8)$ \\
Altered mental status & $34(43.0)$ \\
Visual disturbances & $8(10.1)$ \\
Behavior changes & $7(8.9)$ \\
Seizures & $11(13.9)$ \\
Nuchal rigidity & $15(18.9)$ \\
Cranial nerve palsy & $8(10.1)$ \\
Motor deficit & $8(10.1)$ \\
Cough & $19(24.1)$ \\
Dyspnea & $9(11.4)$ \\
Constitutional symptoms & $21(26.6)$ \\
\hline
\end{tabular}

TABLE 3: Laboratory findings of 79 patients with cryptococcal meningitis.

\begin{tabular}{lccc}
\hline Variable & $\begin{array}{c}\text { Initial } \\
\text { Mean } \\
\text { (range) }\end{array}$ & $\begin{array}{c}{ }^{\mathrm{a}} \text { After } \\
\text { treatment } \\
\text { Mean (range) }\end{array}$ & $\begin{array}{c}p \\
\text { value }\end{array}$ \\
\hline $\begin{array}{l}\text { Opening pressure }(\mathrm{cm} \\
\left.\mathrm{H}_{2} \mathrm{O}\right)\end{array}$ & $30(10-130)$ & $22(10-50)$ & 0.387 \\
$\mathrm{CSF}$ glucose $(\mathrm{mg} / \mathrm{dL})$ & 36.5 & $43(2-154)$ & 0.278 \\
CSF leukocytes $(\mathrm{cells} / \mu \mathrm{L})$ & $25(1-853)$ & $8(0-300)$ & $<0.05$ \\
CSF proteins $(\mathrm{mg} / \mathrm{dL})$ & $82(0-882)$ & $59(0-1866)$ & 0.228 \\
\hline & $(\%)$ & $N(\%)$ & \\
\hline $\begin{array}{l}\text { CSF antigen } \\
\text { CSF positive culture }\end{array}$ & $(55.6)$ & $7(8.8)$ & 0.642 \\
$\begin{array}{l}\text { Blood culture positive } \\
\text { Blood antigen } \geq 1: 1000\end{array}$ & $(93.6)$ & $6(7.5)$ & 1.00 \\
Bronchoalveolar lavage & $(45.5)$ & - & - \\
positive & $(7.5)$ & - & - \\
\hline
\end{tabular}

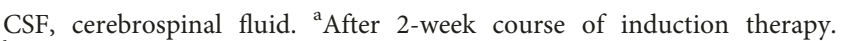
${ }^{\mathrm{b}}$ Cryptococcus antigen. ${ }^{\mathrm{c}}$ Collected within 14-day period of induction therapy.

60 -day mortalities were $19 \%$ and $24 \%$, respectively. Multivariate analysis of Cox regression identified headache at presentation, mechanical ventilation, CSF glucose $<20 \mathrm{mg} / \mathrm{dL}$, and CSF cryptococcal antigen $\geq 1: 1000$ as independent risk factors for mortality.

\section{Discussion}

The incidence of cryptococcal meningitis has increased after the pandemic of AIDS and despite availability of HAART continues to be the most common cause of opportunistic meningitis in many countries [4-6]. Although the number of cases of cryptococcal meningitis diminished in the HAART era, Brazil, Colombia, Mexico, and Argentina are the countries with the highest incidence of meningeal cryptococcosis in Latin America [7]. Although access to HIV diagnosis, universal access, and immediate start to HAART therapy constitutes a major medical
TABLE 4: Comparison between induction treatment schemes of 79 patients with cryptococcal meningitis.

\begin{tabular}{lccc}
\hline Variable & $\begin{array}{c}\text { AmB + 5-FC } \\
\text { Mean (range) }\end{array}$ & $\begin{array}{c}\text { AmB +flu } \\
\text { Mean } \\
\text { (range) }\end{array}$ & $\begin{array}{c}p \\
\text { value }\end{array}$ \\
\hline Time, weeks & 2.65 & $2.6(1-5.43)$ & - \\
AmBd dose & $(0.57-14.4)$ & $52.4(50-90)$ & - \\
LFAmB dose & $50(20.6-69.1)$ & 275 & - \\
& $200(100-371)$ & $(200-375)$ & \\
5-FC dose & 6000 & - & - \\
& $(1280-9800)$ & 800 & - \\
Flu dose & - & $(300-1000)$ & - \\
& $(\%)$ & $(\%)$ & - \\
Hypokalemia & $(67.0)$ & $(15.1)$ & - \\
Hypomagnesemia & $(67.0)$ & $(8.8)$ & - \\
Acute renal injury & $(67.0)$ & $(10.1)$ & - \\
Anemia & $(67.0)$ & $(12.6)$ & - \\
Flu-consolidation & $(51.8)$ & $(15.1)$ & - \\
Time of consolidation, & $8(1.5-8)$ & $7.8(2-8)$ & - \\
weeks & $(11.4)$ & $(26.7)$ & 0.210 \\
30-day mortality & $(17.4)$ & $(26.7)$ & 0.462 \\
60-day mortality &
\end{tabular}

AmB, amphotericin B; 5-FC, flucytosine (mg/day); Flu, fluconazole (mg/day); IQR, interquartile range; AmBd, amphotericin deoxycholate; LFAmb, lipid formulations of amphotericin B. ${ }^{\mathrm{a}}$ Dose reported in $\mathrm{mg} /$ day.

advancement in the clinical management of HIV in Latin American countries such as Brazil, its success depends on strict adherence to prescribed regimen [8]. Approximately $50 \%$ of our patients with meningeal cryptococcosis were on HAART, with probably poor adherence to medical treatment. In a recent study in Brazil, a higher frequency of patients with CD $4+$ cell count $<200$ cells $/ \mathrm{mL}$ was observed in 2014-2015 when compared to the previous years [9]. This suggests that the adopted strategies for early diagnosis of HIV/AIDS are still ineffective. In contrast, with the advent of effective antiretroviral therapy, the majority of cases of cryptococcal meningitis in developed nations occur among non-HIV-infected patients, especially organ transplant recipients [10]. In fact, cryptococcosis is the third most commonly occurring invasive fungal infection, after invasive candidiasis and aspergillosis, in solid organ transplant recipients [11]. It has been observed that cryptococcal meningitis in liver and kidney recipients are late infections in our institution, and considered to represent reactivation of latent infection in the recipient. The median time to cryptococcal disease from transplantation in our study was 382 days (data not shown).

As expected, Cryptococcus neoformans species complex was the predominant species isolated in our patients. $C$. neoformans is globally the major fungal pathogen causing infections in immunocompromised individuals such as AIDS patients, with an estimated incidence of 223,100 cases in 2014 [4]. C. neoformans has long been considered as the culprit causing cryptococcosis in HIV-infected patients including countries in Latin America [12, 13]. The third largest number of cases in the world occurred in Latin America, with an estimated incidence of 5,300 cases per year. 
Brazil and Colombia followed by Argentina and Mexico were the countries with the highest incidence [4].

Despite availability of universal HAART, appropriate antifungal treatment including amphotericin $\mathrm{B}$ and flucytosine/fluconazole, and management of intracranial hypertension in a reference tertiary care hospital according to the IDSA guidelines, our mortality was high (60-day mortality of 25\%) [14]. The median time between diagnosis of cryptococcal meningitis and HAART initiation was 21 days (data not shown). In our institution, we recommend that HAART initiation should be delayed until there is evidence of a sustained clinical response to antifungal therapy. Early initiation of HAART resulting in IRIS has been described in patients with tuberculous meningitis, and the evidence remains mixed for those with cryptococcosis [15]. Additional studies are warranted to provide the evidence base for effective decision making. Considering our approach of delayed initiation of HAART, we observed only five cases of immune reconstitution inflammatory syndrome which did not impact in the interpretation of our results (data not shown).

Cerebrospinal fluid high opening pressure occurred in the majority of our patients and persisted despite adequate antifungal treatment. In fact, the kinetics of release of a pivotal cytokine like TNF-a in immune responses induced by cryptococcal cell envelope components will reflect on the course of the infection, its containment, and ultimately its elimination by phagocytes $[16,17]$. Headache was an independent risk factor for mortality in our study and considered the most common symptom of intracranial hypertension. In addition, CSF glucose levels $<20 \mathrm{mg} / \mathrm{dL}$ and cryptococcal antigen $\geq 1: 1000$ was identified as an important risk factor for death in our cohort. Previous studies have shown that initial high CSF cryptococcal antigen titers were independent predictors of mortality in cryptococcal meningitis, especially in non-HIV-infected patients $[18,19]$. Considering Brazil as a middle-income country by the World Bank (2017), our mortality was lower than the estimated mortality for middle-income countries and similar to other countries in Europe and North America. Rajasingham et al. estimated 1-year mortality in low-income countries as $70 \%$ after cryptococcal meningitis for those in care and $100 \%$ for those not in care. In middle-income countries, the estimated 1-year mortality was $40 \%$ (uncertainty interval 34-46), based on summary statistics of outcomes of those receiving amphotericin B and fluconazole, and $60 \%$ for those not originally in care [4]. In Europe, the estimated 1-year mortality was $30 \%$ for those in care and $45 \%$ for those not in care. In North America, the estimated 1 -year mortality was $20 \%$ for those in care and $30 \%$ for those not in care [4].

In summary, the present study highlights important characteristics of a recent cohort of patients with cryptococcal meningitis attending a tertiary care hospital in Brazil after 23 years of the institution of universal HAART. Cryptococcal meningitis continues to be a prevalent opportunistic infection in HIV-infected patients. In addition, mortality and adverse neurologic sequelae from HIVassociated cryptococcal meningitis remain high due to raised intracranial pressure complications despite appropriate treatment. Identification of risk factors and additional treatment modalities, especially for intracranial hypertension, are necessary to improve care for patients with cryptococcal meningitis. Although cryptococcal disease rates are decreasing over time, the associated mortality and costs remain concerning.

\section{Data Availability}

The data used to support the findings of this study are available from the corresponding author upon request.

\section{Conflicts of Interest}

The authors declare that they have no conflicts of interest.

\section{Acknowledgments}

This study was supported in part by CNPq.

\section{References}

[1] S. M. Levitz, "The ecology of Cryptococcus neoformans and the epidemiology of cryptococcosis," Clinical Infectious Diseases, vol. 13, no. 6, pp. 1163-1169, 1991.

[2] J. R. Perfect and A. Casadevall, "Cryptococcosis," Infectious Disease Clinics of North America, vol. 16, no. 4, pp. 837-874, 2002.

[3] J. N. Jarvis and T. S. Harrison, "HIV-associated cryptococcal meningitis,” AIDS, vol. 21, no. 16, pp. 2119-2129, 2007.

[4] R. Rajasingham, R. M. Smith, B. J. Park et al., "Global burden of disease of $\mathrm{HIV}$-associated cryptococcal meningitis: an updated analysis," The Lancet Infectious Diseases, vol. 17, no. 8, pp. 873-881, 2017.

[5] O. Lortholary, G. Poizat, V. Zeller et al., "Long-term outcome of AIDS-associated cryptococcosis in the era of combination antiretroviral therapy," AIDS, vol. 20, no. 17, pp. 2183-91, 2006.

[6] H. R. Brodt, B. S. Kamps, P. Gute, B. Knupp, S. Staszewski, and E. B. Helm, "Changing incidence of AIDS-defining illnesses in the era of antiretroviral combination therapy," AIDS, vol. 11, no. 14, pp. 1731-1738, 1997.

[7] C. Firacative, J. Lizarazo, M. T. Illnait-Zaragozí, and E. Castañeda, "The status of cryptococcosis in Latin America," Memórias do Instituto Oswaldo Cruz, vol. 113, no. 7, article e170554, 2018.

[8] V. Saraceni, M. M. Cruz, L. M. Lauria, and B. Durovni, "Trends and characteristics of AIDS mortality in the Rio de Janeiro city after the introduction of highly active antiretroviral therapy," Brazilian Journal of Infectious Diseases, vol. 9, no. 3, pp. 209-215, 2005.

[9] M. F. Pereira, E. Luz, E. M. Netto, M. H. F. Barbosa, and C. Brites, "Low variation in initial CD4 cell count in a HIV referral center, in Salvador, Brazil, from 2002 to 2015," Brazilian Journal of Infectious Diseases, vol. 22, no. 3, pp. 245-247.

[10] P. G. Pappas, "Cryptococcal infections in non-HIV-infected patients," Transactions of the American Clinical and Climatological Association, vol. 124, pp. 61-79, 2013.

[11] I. A. George, C. A. Q. Santos, M. A. Olsen, and W. G. Powderly, "Epidemiology of cryptococcosis and cryptococcal meningitis in a large retrospective cohort of 
patients after solid organ transplantation," Open Forum Infectious Diseases, vol. 4, no. 1, pp. S75-S76, 2017.

[12] B. Speed and D. Dunt, "Clinical and host differences between infections with the two varieties of Cryptococcus neoformans," Clinical Infectious Diseases, vol. 21, no. 1, pp. 28-34, 1995.

[13] T. Spina-Tensini, M. D. Muro, F. Queiroz-Telles et al., "Geographic distribution of patients affected by Cryptococcus neoformans/Cryptococcus gattii species complexes meningitis, pigeon and tree populations in Southern Brazil," Mycoses, vol. 60 , no. 1, pp. 51-58, 2017.

[14] J. R. Perfect, W. E. Dismukes, F. Dromer et al., "Clinical practice guidelines for the management of cryptococcal disease: 2010 update by the Infectious Diseases Society of America," Clinical Infectious Diseases, vol. 50, no. 3, pp. 291-322, 2010.

[15] D. R. Boulware, D. B. Meya, C. Muzoora et al., "Timing of antiretroviral therapy after diagnosis of cryptococcal meningitis," New England Journal of Medicine, vol. 370, no. 26, pp. 2487-2498, 2014.

[16] W. Chaka, A. F. Verheul, and A. I. Hoepelman, "Influence of different conditions on kinetics of tumor necrosis factor alpha release by peripheral blood mononuclear cells after stimulation with Cryptococcus neoformans: a possible explanation for different results," Clinical Vaccine Immunology, vol. 4, no. 6, pp. 792-794, 1997.

[17] W. Chaka, A. F. Verheul, V. V. Vaishnav et al., "Cryptococcus neoformans and cryptococcal glucuronoxylomannan, galactoxylomannan, and mannoprotein induce different levels of tumor necrosis factor alpha in human peripheral blood mononuclear cells," Infection and Immunity, vol. 65, no. 1, pp. 272-278, 1997.

[18] C. C. Shih, Y. C. Chen, S. C. Chang, K. T. Luh, and W. C. Hsieh, "Cryptococcal meningitis in non-HIV-infected patients,” QJM, vol. 93, no. 4, pp. 245-51, 2000.

[19] C. H. Liao, C. Y. Chi, Y. J. Wang et al., "Different presentations and outcomes between HIV-infected and HIV-uninfected patients with Cryptococcal meningitis," Journal of Microbiology, Immunology and Infection, vol. 45, no. 4, pp. 296-304, 2012. 


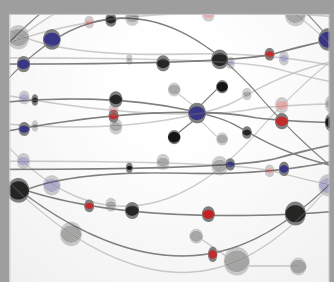

The Scientific World Journal
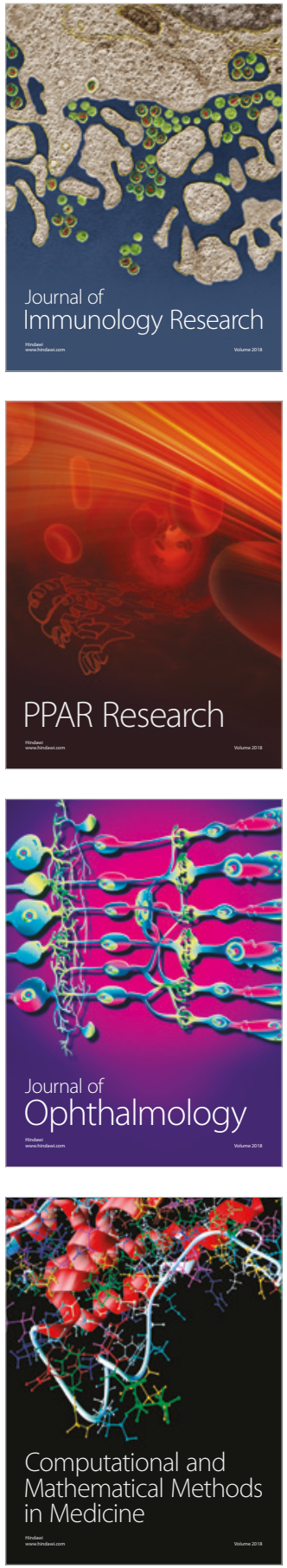

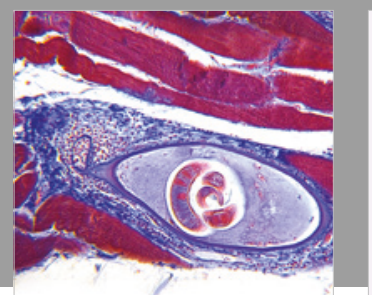

Gastroenterology Research and Practice

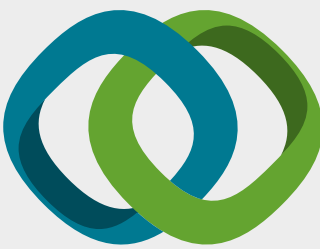

\section{Hindawi}

Submit your manuscripts at

www.hindawi.com
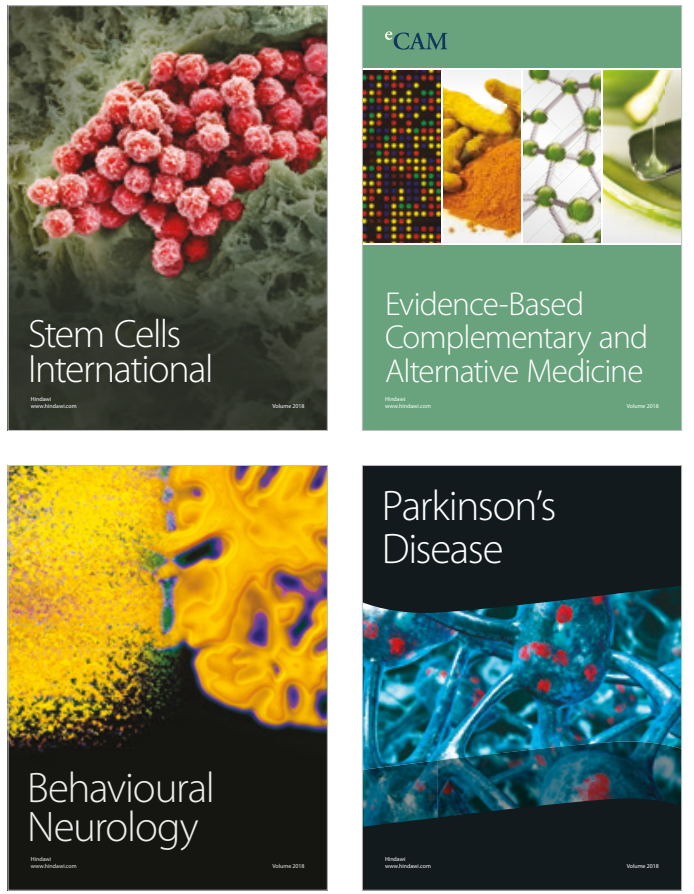

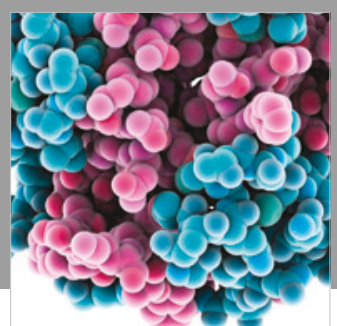

ournal of

Diabetes Research

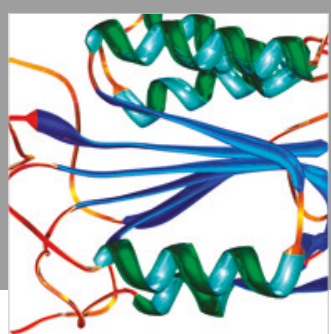

Disease Markers
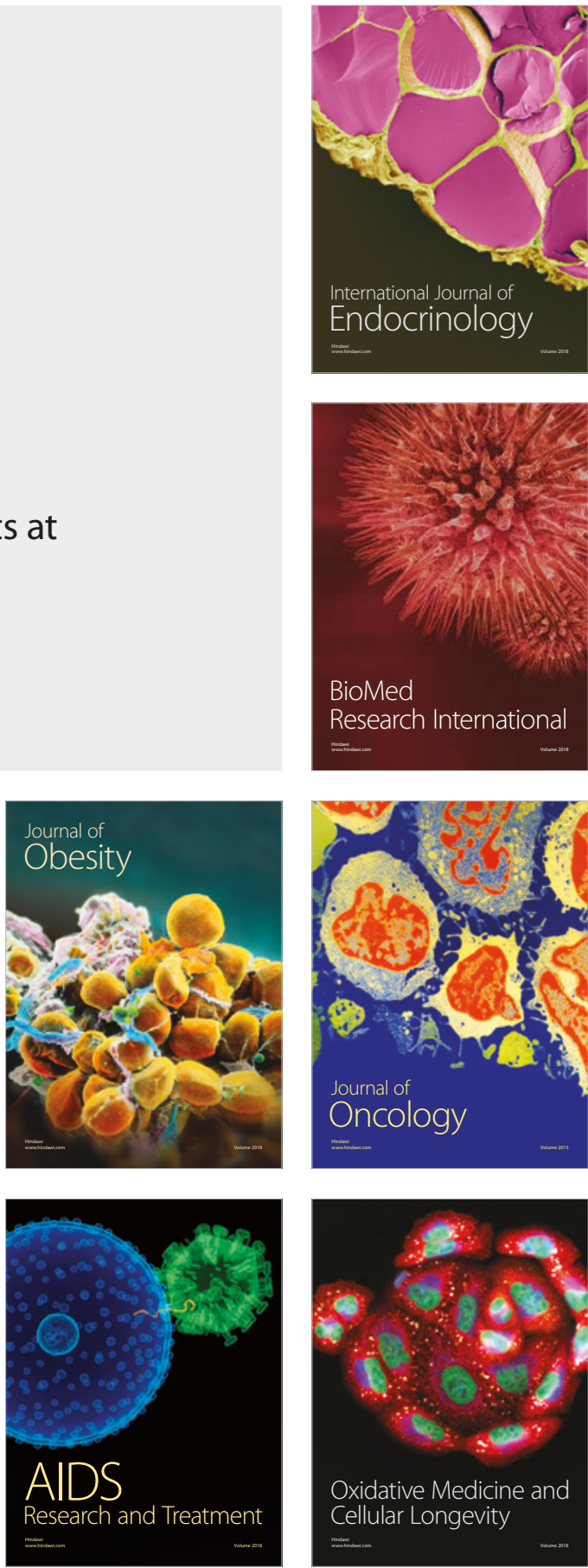